\title{
Anisotropy, Geometric Structure and Frustration Effects in Molecule-Based Nanomagnets
}

\author{
G. Kamieniarz ${ }^{a}, *$ P. KozŁowski ${ }^{a}$, M. Antkowiak ${ }^{a}, \mathrm{P}$ Sobczak $^{a}$, T. Ślusarski $^{a}$, \\ D.M. TomeckA ${ }^{a}$, A. Barasiński ${ }^{b}$, B. Brzostowski ${ }^{b}$, A. Drzewiński $^{b}$, A. Bieńko $^{c}$ \\ AND J. MROZIŃSKI ${ }^{c}$ \\ ${ }^{a}$ Faculty of Physics, A. Mickiewicz University, Umultowska 85, 61-614 Poznań, Poland \\ ${ }^{b}$ Institute of Physics, University of Zielona Góra, Poland \\ ${ }^{c}$ Faculty of Chemistry, University of Wrocław, Poland
}

\begin{abstract}
In this paper we review our results of comprehensive study of molecular nanomagnets recently synthesized in the form of the chromium-based molecules and bimetallic copper-containing chains as well as we present some new findings. We focus on effects of anisotropy, geometry and frustration appearing in various thermodynamic properties of the nanoscale magnets which are described by Heisenberg-like spin models and simulated by accurate numerical methods. We show that bond-dependent exchange anisotropy is needed to model magnetic torque in the Cd-doped chromium ring. We argue that only in the limit of infinite rings $(n \rightarrow \infty)$ frustration can be considered as the opposite to bipartiteness in the odd numbered $(3 \leq n \leq 9) s=3 / 2$ quantum spin rings. We analyse the influence of exchange interactions and anisotropy on magnetic susceptibility of bimetallic $(S=3 / 2$, $s=1 / 2$ ) chains composed of $\mathrm{Cu}$ ions linked to different $3 d$ ions by fitting experimental data. We reach the remarkable consistency of the density functional theory estimates of the magnetic couplings in $\mathrm{Cr}_{8}$ molecule and provide strong support to the spin models exploited in the literature.
\end{abstract}

PACS: 75.50.Xx, 75.10.Jm, 75.10.Pq, 75.40.Cx

\section{Introduction}

Molecular magnets, which are examples of nanoscale magnetic systems have been recently objects of intensive research [1]. Their magnetic properties are determined mainly by transition metal ions embedded in molecular hosts. Due to effective shielding caused by molecular ligands magnetic interactions between metal ions are significant only within a single molecule. Thus, a practical absence of inter-molecular and presence of strong intra-molecular magnetic interactions give rise to interesting quantum effects such as resonant tunneling of magnetization [2] or tunneling of the Néel vector [3]. Slow relaxation of magnetization caused by strong anisotropy makes such systems good candidates for application in dense storage devices [4]. Another promising area of their envisaged applications is quantum computing [5-8].

Properties of particular molecular magnets are determined by many factors. Obviously a spin value of transition metal ions is very important. Dominating exchange interactions between the ions can be ferro- (like in $\mathrm{Ni}_{12}$ $[9,10]$ ) or antiferromagnetic (like in $\mathrm{Mn}_{12}$ [11]) leading to quite different ground states and magnetic properties.

\footnotetext{
* corresponding author; e-mail: gjk@amu.edu.pl
}

The molecules with a large value of the total spin and single-ion anisotropy are called single molecule magnets (SMM) as they behave as small nanoscale magnets [11]. Anisotropy is determined by the type of the metal ions and composition and symmetry of the ions' neighborhood. The strength of the interactions depends on the sort and number of bridging ligands [12]. Besides, the overall topology of the molecule strongly influences its properties $[13,14]$. Molecules doped with other ions, or containing broken bonds exhibit quite different properties than their pure counterparts [15]. It has been demonstrated [5] that even the type of hydrogen isotope present in the ligands can strongly influence relaxation time.

The physics of molecular magnets can be usually well described by the quantum Heisenberg model with single-ion and exchange anisotropy terms

$$
\begin{aligned}
\mathcal{H} & =\sum_{j=1}^{n-1}\left(J s_{j} \cdot s_{j+1}+s_{j} \cdot R_{j} D_{\mathrm{e}} R_{j}^{-1} s_{j+1}\right)+\alpha J s_{n} \cdot s_{1} \\
& +\sum_{j=1}^{n}\left[D\left(s_{j}^{z}\right)^{2}-g \mu_{\mathrm{B}} B\left(s_{j}^{z} \cos \theta+s_{j}^{x} \sin \theta\right)\right] .
\end{aligned}
$$

Here $s_{j}$ stands for a spin operator located on site $j, D$ is the single ion anisotropy and $D_{\mathrm{e}}$ stands for the exchange anisotropy tensor defined in the local coordinate system. 
$R_{j}$ are the rotation matrices used to transform tensor $D_{\mathrm{e}}$ into the global coordinate system. With the exception of $D_{\mathrm{e}}$ all the elements of the Hamiltonian (1) are expressed in the global coordinate system and $\theta$ stands for the angle between magnetic field (applied in the $x-z$ plane) and the $z$ axis. The parameter $\alpha$ determines the topology of a given spin system. For $\alpha=0$ we have a segment with free boundary conditions, for $\alpha=1$ - a uniform ring whereas for $\alpha \neq 1$ - a bond defect in the ring.

This model in general is not integrable so that the calculations are then performed by means of various numerical methods. Comparison with different experiments enables determination of all the model parameters. The additional verification of the assumed spin model and the values of the parameters can be done by the density functional theory (DFT) calculations.

In this paper we review some of our recent results focusing on a few factors determining magnetic properties of molecular magnets, such as anisotropy, geometry and often resulting frustration, as well as we render some new results. In particular we discuss anisotropy in the chromium-doped rings [16-18], geometric frustration effects in homometallic nanonuclear $s=3 / 2$ rings [15] and present new results for smaller odd numbered rings needed to perform some extrapolations, and supplement the DFT results for the precursor of the chromium ring family $[19,20]$, filling up the set of the non-equivalent spin configurations. Finally the results for the magnetic chains with alternating two different metal ions (Co, Mn, $\mathrm{Ni}, \mathrm{Re}$ and $\mathrm{Cu}$ ) are presented [21] with emphasis on the new analysis of the $\operatorname{Re}(\mathrm{IV})$ compound bridged by the chlorine ions [22].

To perform calculations for Heisenberg-like Hamiltonians we used three numerical techniques: quantum transfer matrix [23], exact diagonalization (ED) [10], and density matrix renormalization group (DMRG) [24, 25]. The DFT calculations were performed within the pseudopotential approach, using the Siesta package [26].

\section{Chromium-based rings}

Among molecular rings those derived from the precursor $\left[\mathrm{Cr}(\mathrm{III})_{8} \mathrm{~F}_{8}\left(\mathrm{Me}_{3} \mathrm{CCOO}\right)_{16}\right]\left(\mathrm{Cr}_{8}\right.$ in short) [27] form especially rich and interesting family and attracted a lot of interest $[28,29]$. In the rings $\mathrm{Cr}$ ions with spin $S=3 / 2$ are coupled by antiferromagnetic nearest neighbor intramolecular exchange interactions. They can also be doped with other metal ions [29], can form dimers [7] and contain even or odd number of magnetic centers [30].

\subsection{Anisotropy in $\mathrm{Cr}_{7} \mathrm{Cd}$}

In the heterometallic chromium-based molecular ring $\left[\mathrm{Me}_{2} \mathrm{NH}_{2}\right]\left[\mathrm{Cr}(\mathrm{III})_{7} \mathrm{Cd}(\mathrm{II}) \mathrm{F}_{8}\left(\mathrm{Me}_{3} \mathrm{CCOO}\right)_{16}\right] \quad\left(\mathrm{Cr}_{7} \mathrm{Cd}\right.$ in short) the controversy concerned the type and magnitude of anisotropy. $\mathrm{Cr}_{7} \mathrm{Cd}$ is a member of the $\mathrm{Cr}_{8}$ family where one of the $\mathrm{Cr}$ ions was substituted by $\mathrm{Cd}[31]$. It is assumed that the properties of $\mathrm{Cr}_{7} \mathrm{Cd}$ can be well described by Hamiltonian (1), where $n=7, \alpha=0$, $g=1.98$ and $D_{\mathrm{e}}$ tensors have the same diagonal form [32]: $D_{\mathrm{e}}^{x x}=D_{\mathrm{e}}^{y y}=-D_{\mathrm{e}}^{z z} / 2=-d$. The value of $D_{\mathrm{e}}^{z z}$ is dominating due to a contribution from dipolar spin-spin interactions [32].

We have demonstrated the ambiguity in the fit of the powder-sample susceptibility which can be equally well simulated with three different sets of parameters: I with only single ion anisotropy $(d=0, D=-0.31 \mathrm{~K}$, $J=16.01 \mathrm{~K}$ ) [16], II with single ion and global exchange anisotropies $\left(R_{j}=1, d=-0.0965 \mathrm{~K}, D=-0.209 \mathrm{~K}\right.$, $J=15.82 \mathrm{~K})[16]$ and III with single ion and bond-dependent exchange anisotropies $\left(R_{j}\right.$ depends on $j, d=$ $-0.1525 \mathrm{~K}, D=-0.1928 \mathrm{~K}, J=16.604 \mathrm{~K}$ ) [17]. However, the EPR spectroscopic studies suggested that the third (III) set of parameters should be preferred [32].

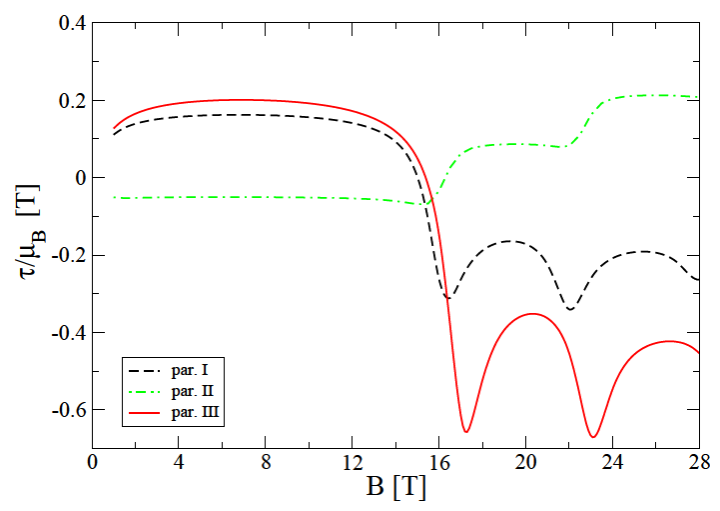

Fig. 1. Magnetic torque of $\mathrm{Cr}_{7} \mathrm{Cd}$ for $\theta=5.5^{\circ}$ at temperature $T=0.4 \mathrm{~K}$.

To determine the right anisotropy also on the basis of magnetic measurements we have extended calculations to a number of thermodynamic quantities for the above mentioned three sets of parameters [16-18]. It appeared that only the simulation of the magnetic torque required the introduction of bond-dependent exchange anisotropy. For other quantities such as global and local magnetization, specific heat and entropy the same or very similar results have been obtained for all three sets of parameters [16-18]. In Fig. 1, which combines results from Refs. $[16,18]$ the magnetic torque is presented as a function of magnetic field. Only qualitative comparison with experiment for magnetically identical compound $\mathrm{Cr}_{7} \mathrm{Zn}$ is possible because the results of the measurements are given in arbitrary units [13]. The shape of the curve obtained for parameters III is the most similar to that obtained in the experiment [13].

This allows us to confirm on the independent ground of thermodynamic measurements the preference for the third set of parameters which includes bond-dependent exchange anisotropy suggested by the EPR studies [32].

\subsection{Frustration in the models of $\mathrm{Cr}_{n}$ rings}

It has been long believed that odd membered antiferromagnetic rings are hard (if not impossible) to synthesize. 
Yet, recently a number of such rings, both homo- and heterometallic were reported $[30,33,34]$. They are especially interesting because of magnetic frustration which is expected to appear in this kind of materials.

Magnetic frustration is an interesting and not precisely defined phenomenon, which was analyzed in the context of both classical [35] and quantum spin systems [36]. Recently frustration effects have been investigated in nanoscale systems [37] and attempts were made to reconcile different approaches to magnetic frustration [38].

In antiferromagnetic spin rings frustration can be caused by competing nearest and next nearest neighbor interactions or by the very geometry of the system containing odd number of spins. In chromium rings next nearest neighbor interactions are negligible (see Sect. 2.2) and thus frustration may be only caused by the geometry of a system.

To investigate the conditions under which frustration may appear in hypothetical odd membered chromium rings we analyzed the models of quantum $s=3 / 2$ spin rings with one broken bond described by Hamiltonian (1), imposing $n=9, \theta=0, D_{\mathrm{e}}=0$. It is assumed that $g=1.98$, coupling $J=-16.6 \mathrm{~K}$ and $D=-0.34 \mathrm{~K}$, which are typical values for chromium rings [16, 17, 32].

Frustration effects are expected for $\alpha>0$ as then the system is not bipartite [38-40]. However, calculating the ground-state phase diagram we have demonstrated [15] that in the model of $\mathrm{Cr}_{9}$ frustration appears for $\alpha>\alpha_{\mathrm{c}}(B)>0$. In the frustrated phase the ground state is the $m=1 / 2$ doublet $(M= \pm m)$, whereas in the non-frustrated phase the ground state is the $m=3 / 2$ doublet. This change in the ground state is manifested by an abrupt change of local and global frustration signatures defined and calculated at $T=1 \mathrm{~K}$ in Ref. [15].

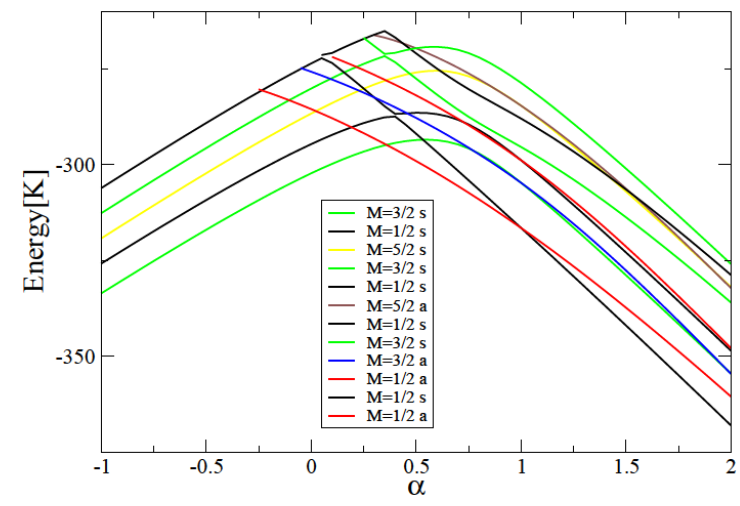

Fig. 2. Low-lying energy levels of $\mathrm{Cr}_{7}$ versus $\alpha$ for $B=5 \mathrm{~T}$.

Similar effects are also expected for smaller and larger odd numbered rings. In Fig. 2 the energy structure of $\mathrm{Cr}_{7}$ is presented. Like for $\mathrm{Cr}_{9}$ [15], at a certain value of $\alpha_{\mathrm{c}}=0.36$ the ground state of the system is changed. Here again the $m=1 / 2$ doublet corresponds to the frustrated phase and the $m=3 / 2$ one to the non-frustrated. The second intersection of the lowest lying states takes place

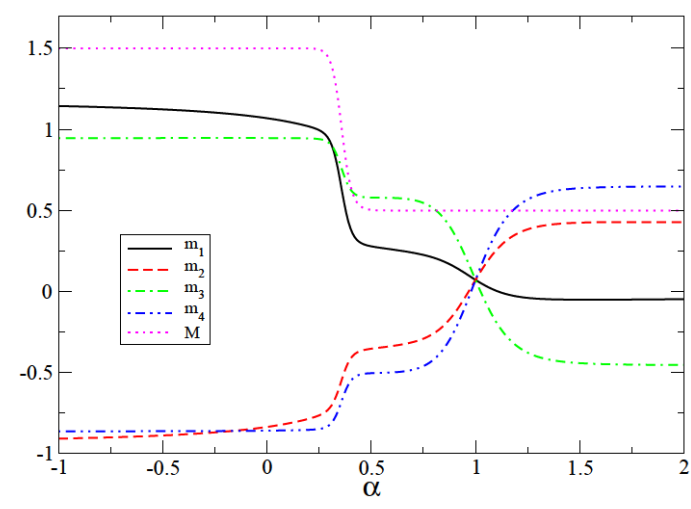

Fig. 3. Local $\left(m_{j}\right)$ and global $(M)$ magnetizations of $\mathrm{Cr}_{7}$ as functions of $\alpha$ for $T=1 \mathrm{~K}$ and $B=5 \mathrm{~T}$.

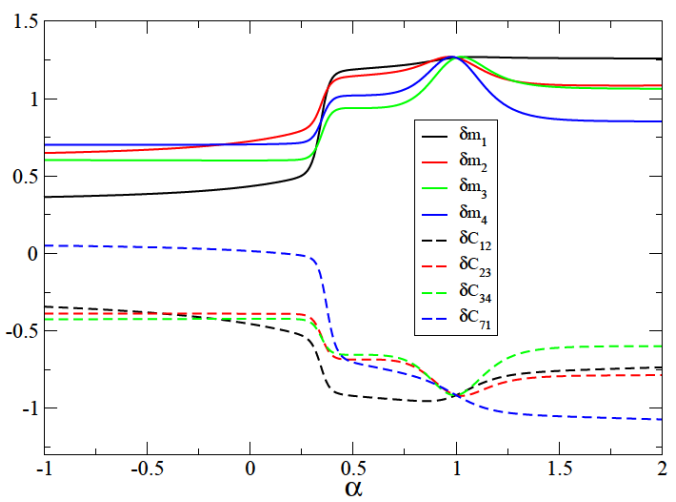

Fig. 4. Local fluctuations and correlations of $\mathrm{Cr}_{7}$ as functions of $\alpha$ for $T=1 \mathrm{~K}$ and $B=5 \mathrm{~T}$.

at $\alpha=1$ and is related to the change of the symmetry but the ring remains still frustrated.

The frustration signatures in the $\mathrm{Cr}_{7}$ ring undergo abrupt changes at the value of $\alpha$ corresponding to the transition from the frustrated to the non-frustrated phase. Stronger frustration corresponds to the reduction of the local and global magnetizations (Fig. 3) and increase of the local fluctuations (Fig. 4).

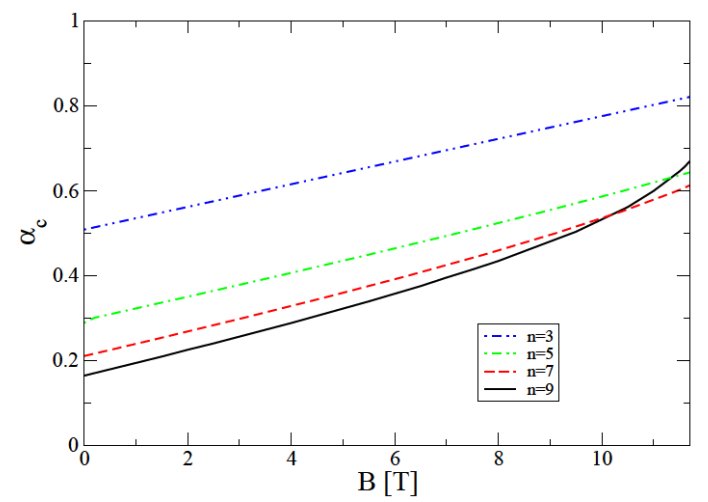

Fig. 5. $\alpha_{\mathrm{c}}$ as a function of magnetic field for various ring sizes. 


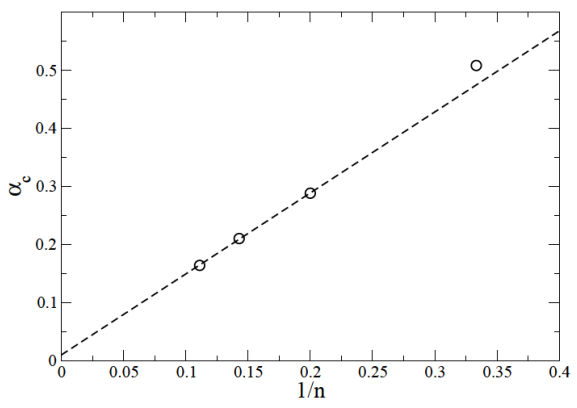

Fig. 6. $\alpha_{\mathrm{c}}$ for the magnetic field $B=0$ as a function of $1 / n-$ circles. The regression line for the three largest rings is marked by a broken line.

The results obtained for the odd numbered rings $(n=$ $3,5,7,9)$ allow us to plot the ground state phase diagram presenting the dependence of $\alpha_{\mathrm{c}}$ on the size of the system and the magnetic field (Fig. 5). Above $\alpha_{\mathrm{c}}$ the corresponding systems are frustrated (the ground state $m=1 / 2$ ), below they are not frustrated (the ground state $m=3 / 2$ ).

For $B=0$ extrapolation of $\alpha_{\mathrm{c}}(1 / n)$ for finite $n=5,7,9$ and $n=7,9$ to infinite size (see Fig. 6 ) gives values $\alpha_{\mathrm{c}}(0)=0.0098$ and 0.0036 , respectively, which are very close to zero. This result allows us to conclude that only for the infinite size odd numbered $s=3 / 2$ rings the conjecture of Schnack [38] stating that bipartiteness can be considered as the opposite to frustration, may be valid.

\subsection{DFT results for $\mathrm{Cr}_{8}$}

In order to extract the electronic structure as well as to estimate exchange integrals and local spin densities in $\mathrm{Cr}_{8}$ beyond the spin models, we performed the DFT calculations [20] based on the pseudopotential approach implemented in the Siesta package [26]. Previously we checked that [19] the approach is reliable despite it is computationally less demanding than the codes with the full potential implemented. Instead of using various linear or zig-zag models [41, 42], we have accounted for a real geometrical structure of the molecule (like that used in [43]) and have evaluated the total energies for all the non-equivalent configurations with spin up and down.

The 15 differences in the total energies of 16 spin configurations previously calculated [20] relative to the fully antiferromagnetic ground state, are supplemented by the two remaining nonequivalent configurations

$$
--+-+-++; \quad--+-++-+
$$

yielding the energy differences $\Delta E[\mathrm{meV}]$ equal to 64.066 and 64.136 , respectively.

By comparing all possible combinations of these 17 DFT energy differences with those of the Ising models, we have estimated the exchange integrals $J$ between chromium ions, using the topology of the two-spin interactions plotted in Fig. 7. The exchange interaction parameters obtained are presented in Table I. We tried

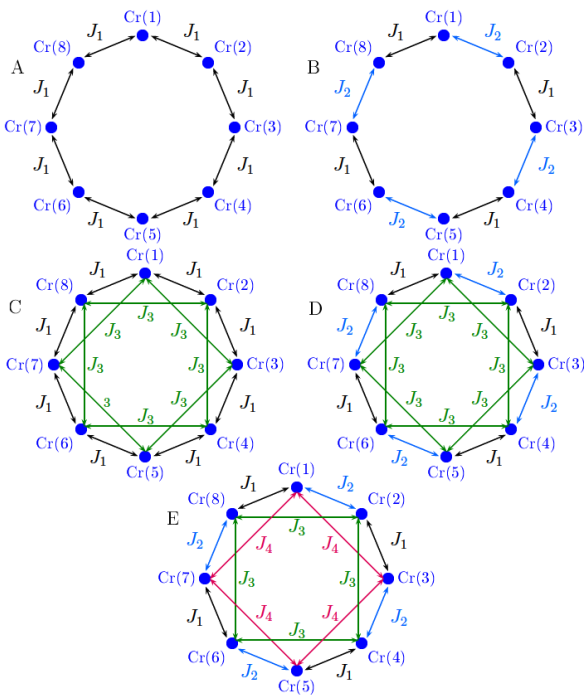

Fig. 7. Schematic representation of the Ising models considered. The bonds $J_{1}, J_{2}$ and $J_{3}, J_{4}$ denote the nearest- and the next-nearest neighbour couplings, respectively.

a number of possibilities and found out that the width of the estimates decreases with increasing number of parameters.

\section{TABLE I}

The exchange couplings following from the models A-E. The columns $J$ and $J^{\prime}$ contain the medians of the nearest-neighbour and the next nearest-neighbour couplings, respectively (in meV). The values denoted $\mathrm{A}_{f}-\mathrm{E}_{f}$ proceed from the fitting procedure.

\begin{tabular}{l|c|c|c|c|c|c|c}
\hline \hline & $\mathrm{NoD}$ & $J$ & $J_{\min }$ & $J_{\max }$ & $J^{\prime}$ & $J_{\min }^{\prime}$ & $J_{\max }^{\prime}$ \\
\hline $\mathrm{A}$ & 113 & 6.60 & 5.61 & 7.58 & & & \\
$\mathrm{~A}_{f}$ & & 6.72 & & & & & \\
$\mathrm{~B}$ & 497 & 6.60 & 6.46 & 6.72 & & & \\
$\mathrm{~B}_{f}$ & & 6.63 & & & & & \\
$\mathrm{C}$ & 547 & 6.60 & 4.99 & 8.20 & -0.26 & -0.88 & 0.36 \\
$\mathrm{C}_{f}$ & & 6.57 & & & -0.19 & & \\
$\mathrm{D}$ & 1854 & 6.60 & 6.57 & 6.63 & -0.06 & -0.09 & -0.03 \\
$\mathrm{D}_{f}$ & & 6.60 & & & -0.06 & & \\
$\mathrm{E}$ & 3865 & 6.60 & 6.58 & 6.61 & -0.06 & -0.08 & -0.03 \\
$\mathrm{E}_{f}$ & & 6.60 & & & -0.06 & &
\end{tabular}

The parameters evaluated from the model $\mathrm{B}$ cover more narrow interval than those from the model $\mathrm{A}$. The same is noticed for the models C, D and E. The best strategy is to take an average of the nearest neighbour couplings $J_{1}$ and $J_{2}$ (and the next nearest neighbour couplings $J_{3}$ and $J_{4}$ ) calculated from the models B, $\mathrm{D}$ and $\mathrm{E}$. The estimates of the nearest neighbour couplings $J$ (and the next nearest neighbour $J^{\prime}$ ) for the models $\mathrm{B}, \mathrm{D}$ and $\mathrm{E}$ given in Table I proceed from the averaging $J=\left(J_{1}+J_{2}\right) / 2$ and $J^{\prime}=\left(J_{3}+J_{4}\right) / 2$.

In Table I the medians of the nearest neigbhour coupling $J$ and the next nearest neigbour coupling $J^{\prime}$ are presented. The number of data (NoD) is shown in the 
second column. The abbreviations "min" and "max" in the corresponding columns of Table I determine the range of the values calculated for $J$ and $J^{\prime}$.

The medians are very stable and the width of the estimates is decreasing from 3.21 to $0.03 \mathrm{meV}$ for $J$ and from 1.24 to $0.05 \mathrm{meV}$ for $J^{\prime}$. The small values $J^{\prime}$ with respect to $J$ found for the models B, D and E justify omission of this interaction in the spin models introduced in the previous sections.

For all the seventeen DFT energy differences and their spin model counterparts we have performed the least-squares fit with respect to the exchange parameters. The results of the optimization procedure (after averaging in the case of the models B, D, E) are listed in Table I and are denoted $\mathrm{A}_{f}-\mathrm{E}_{f}$.

The present values of the couplings are very consistent and agree remarkably well with those found for a limited number of configurations [19, 20,43]. They give the sound evidence of consistency of the DFT calculations.

Our calculations show consistency in the DFT estimates of the magnetic couplings but do not eliminate inconsistency with experiment. The values of the nearest neighbour coupling overestimate by a factor of 4 the results obtained by fitting experimental data with the predictions of spin models $[16,32,44]$. This deficiency is inherent, happens also in other DFT studies [43,41] and needs further studies.

Another important property that can be calculated by DFT method to verify Heiseberg-like spin models used for chromium rings is the spin polarization of the charge density and the local magnetic moment. As expected, significant values of the spin polarisation can be observed only in the vicinity of the chromium ions [20] which confirms the localized picture assumed in the spin models.

\section{Quantum spin chains}

An interesting class of low-dimensional magnets are the single chain magnets (SCM). They have usually a form of polymer chains with built-in magnetic ions. Strong Ising-like anisotropy and weak inter-chain magnetic interactions give rise to slow relaxation and hysteresis loops of molecular origin, the behaviour observed also in SMM $[1,45]$. In some circumstances such systems can be considered as one-dimensional and modeled with quantum Heisenberg-like models (1). However, for macroscopic $n$ the simulations are computationally very demanding in the low-temperature region.

In our recent papers $[21,24,25,46]$ we have demonstrated that the quantum heterobimetallic chains with the alternating spin values $S=1 / 2$ and $S=3 / 2$ can efficiently model the compounds containing $\mathrm{Cu}(\mathrm{II})$ ions coupled to some $3 d$ ions by thiocyanate bridges in a number of coordinations or to $5 d$ ions $\operatorname{Re}(\mathrm{IV})$ which are known of their strong anisotropy. The bridging thiocyanate ligands are not common. In the literature only a few examples of heterometallic compounds of the type $\mathrm{Cu}-\mathrm{NCS}-\mathrm{M}$ $(\mathrm{M}=\mathrm{Cr}(\mathrm{III}), \mathrm{Co}(\mathrm{II}), \mathrm{Mn}(\mathrm{II}))$ have been synthesized.
The thiocyanide bridges are less efficient in transmitting the magnetic interactions between metal ions so that the interactions may become weaker than the value of the corresponding single-ion anisotropy. This implies the need for accurate account of the anisotropy effects.

In the comprehensive DMRG studies we have simulated the thermodynamic properties of the bimetallic chains and estimated the magnetic couplings and single-ion anisotropy parameters, assuming $D_{\mathrm{e}}=\alpha=\theta=0$ in Eq. (1). The results are summarized in Table II.

TABLE II

The model parameters for the heterobimetallic chains (metal = Co, Mn, Ni, Re).

\begin{tabular}{c|c|c|c|c}
\hline \hline Compound & $J / k_{\mathrm{B}}[\mathrm{K}]$ & $D / k_{\mathrm{B}}[\mathrm{K}]$ & $g_{\mathrm{Cu}}$ & $g_{\text {Metal }}$ \\
\hline $\mathrm{Cu}-\mathrm{Co}$ & $-1.0 \pm 0.1$ & $4.6 \pm 0.6$ & $2.12 \pm 0.04$ & $2.25 \pm 0.04$ \\
$\mathrm{Cu}-\mathrm{Mn}$ & $-0.20 \pm 0.05$ & $0.0 \pm 0.04$ & $2.07 \pm 0.05$ & $1.98 \pm 0.05$ \\
$\mathrm{Cu}-\mathrm{Ni}$ & $0.15 \pm 0.05$ & $9.7 \pm 0.2$ & $2.03 \pm 0.04$ & $2.14 \pm 0.01$ \\
$\mathrm{Cu}-\mathrm{Re} 1$ & $3.5 \pm 0.5$ & $35 \pm 5$ & $2.07 \pm 0.05$ & $1.73 \pm 0.01$ \\
$\mathrm{Cu}-\mathrm{Re} 2$ & $9.5 \pm 0.5$ & $20 \pm 5$ & $2.06 \pm 0.05$ & $1.85 \pm 0.01$
\end{tabular}

We emphasize that we have simulated all these systems irrespective of the type of magnetic interactions and the strength of the single-ion anisotropy [21, 24]. The last item in Table II denoted Cu-Re2 has not been published yet and refers to the bimetallic chain complex $[\mathrm{Cu}($ tren $)] \mathrm{ReCl}_{6} \cdot \mathrm{CH}_{3} \mathrm{OH}$, where tren [tris (2-aminoethyl)amine] is a tetradentate ligand [22].

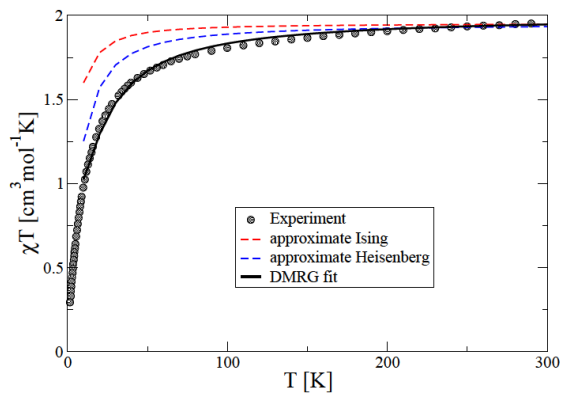

Fig. 8. Susceptibility of the $\mathrm{Cu}-\mathrm{Re} 2$ bimetallic chain. The broken lines show the DMRG results obtained for Ising and Heisenberg models with the parameters estimated earlier in [22]. The continuous line represents our improved fit.

The experimental magnetic data obtained for powder samples were initially fitted with the approximate formula for susceptibility [22] following from the Ising model, giving the exchange coupling $J=3.3 \mathrm{~K}$, the anisotropy constant $D=18.9 \mathrm{~K}$ and $g$-factors $g_{\mathrm{Cu}}=2.11$ and $g_{\mathrm{Re}}=1.81$. We used these values and the DMRG approach to calculate accurately the powder sample susceptibility for the Ising and Heisenberg models. The results are presented as broken lines in Fig. 8 and are quite different from experimental data and the previous approximate calculations fitting them.

Therefore, in order to find a better fit unbiased by any approximation, we reanalyzed the experimental results in 
terms of the model (1). From the fit illustrated in Fig. 8 it has been confirmed that the exchange interactions between the ions are antiferromagnetic but $J=9.5 \pm 0.5 \mathrm{~K}$. Since the measurements were carried out on a powder sample only absolute value of the anisotropy parameter could be determined. Our finding $|D|=20 \pm 5 \mathrm{~K}$ is surprisingly close to the previous estimate [22].

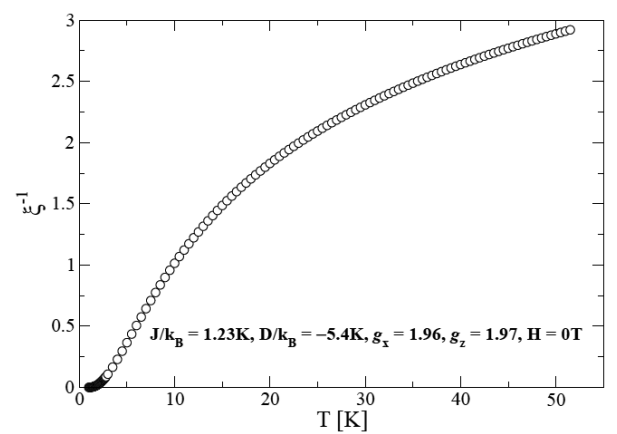

Fig. 9. The temperature dependence of the zero-field inverse correlation length. The plot corresponds to the parameters defined in the legend.

Among SCMs the homometallic compound made of aligned acetate mesotetraphenylporphyrin complexes $\left[\mathrm{Mn}(\mathrm{TPP}) \mathrm{O}_{2} \mathrm{PHPh}\right]-\mathrm{H}_{2} \mathrm{O}$ deserves special attention [45]. Due to the presence of Jahn-Teller effect, $\mathrm{Mn}$ (III) ions $(S=2)$ possess a strong uniaxial anisotropy and the anisotropy axes are arranged alternately along the chain. Using the quantum Heisenberg model which takes into account the complex geometrical structure and the accurate DMRG approach we have demonstrated, despite relatively high spin value, the importance of quantum effects previously neglected in the thermodynamic behaviour [46]. Having found the non-magnetic region in the field-dependent magnetization, we suggested the existence of the singlet Haldane phase which is not correct. The temperature dependence of the correlation length $\xi$ related to the energy gap shown here in Fig. 9 disagrees with this suggestion. The divergence of $\xi$ in the zero-temperature limit rules out the finite-energy gap and is a signature of the Néel phase.

\section{Conclusions}

We have reviewed our recent work on molecular magnets focusing on anisotropy, geometry and frustration effects in ring-shaped and chain like molecule-based nanomagnets. We have analyzed the influence of a bond-dependent exchange anisotropy in $\mathrm{Cr}_{7} \mathrm{Cd}$ on many thermodynamic quantities. For magnetic torque such influence is well pronounced and this type of anisotropy is needed to reach better modeling of this quantity.

For the bimetallic rhenium-copper and the thiocyanate bridged chains the DMRG-based simulations have been carried out for the quantum anisotropic models yielding the estimates of the model parameters. We have established that usually strong anisotropy in comparison to the exchange coupling is responsible for the magnetic properties of these compounds. Contrary to our suggestion, we have shown here that for the canted homometallic Mn(III)-based SCM the Haldane gap cannot be observed.

Within the DFT approach we have accurately evaluated the energies of two missing configurations for the $\mathrm{Cr}_{8}$ molecule and conducted comprehensive analyses of the exchange couplings referring to a set of spin models. Using complementary approach based on the statistical and optimization methods, we have given the strong evidence for the consistency of the DFT results for the nearest- and the next nearest neighbour interactions.

The relation between the topology of the $\mathrm{Cr}_{n}$ rings and frustration effects was analyzed. It was found that in $s=$ $3 / 2$ quantum spin rings only in the limit of infinite size of the ring frustration can be considered as the opposite to bipartitness. For finite odd numbered rings there is a region in the parameter space $(B, \alpha)$ where the system is not bipartite neither frustrated. Our new results for the smaller odd membered rings qualitatively confirm those found earlier for $n=9$.

\section{Acknowledgments}

We acknowledge a support from the MNiSW grant No. 230137 and an access to the platforms of PSNC in Poznan (Poland), TASK in Gdańsk (Poland) and to the PRACE Research Infrastructure resource CURIE based at TGCC (France).

\section{References}

[1] D. Gatteschi, R. Sessoli, J. Villain, Molecular Nanomagnets, Oxford University Press, Oxford 2006.

[2] J.R. Friedman, M.P. Sarachik, J. Tejada, R. Ziolo, Phys. Rev. Lett. 76, 3830 (1996).

[3] P. Santini, S. Carretta, G. Amoretti, T. Guidi, R. Caciuffo, A. Caneschi, D. Rovai, Y. Qiu, J.R.D. Copley, Phys. Rev. B 71, 184405 (2005).

[4] M. Mannini, F. Pineider, P. Sainctavit, C. Danieli, E. Otero, C. Sciancalepore, A.M. Talarico, M.-A. Arrio, A. Cornia, D. Gatteschi, R. Sessoli, Nature Mater. 8, 194 (2009).

[5] A. Ardavan, O. Rival, J.J.L. Morton, S.J. Blundell, A.M. Tyryshkin, G.A. Timco, R.E.P. Winpenny, Phys. Rev. Lett. 98, 057201 (2007).

[6] J. Lehmann, A. Gaita-Ariño, E. Coronado, D. Loss, Nature Nanosci. 2, 312 (2007).

[7] G.A. Timco, S. Carretta, F. Troiani, F. Tuna, R.J. Pritchard, C.A. Muryn, E.J.L. McInnes, A. Ghirri, A. Candini, P. Santini, G. Amoretti, M. Affronte, R.E.P. Winpenny, Nature Nanosci. 4, 173 (2009).

[8] B. Georgeot, F. Mila, Phys. Rev. Lett. 104, 200502 (2010).

[9] A.J. Blake, C.M. Grant, S. Parsons, J.M. Rawson, R.E.P. Winpenny, J. Chem. Soc., Chem. Commun., 2363 (1994) 
[10] A.C. D'Auria, U. Esposito, F. Esposito, G. Kamieniarz, R. Matysiak, J. Phys., Condens. Matter. 13, 2017 (2001).

[11] A. Caneschi, D. Gatteschi, C. Sangregorio, R. Sessoli, L. Sorace, A. Cornia, M.A. Novak, C. Paulsen, W. Wernsdorfer, J. Magn. Magn. Mater. 200, 182 (1999).

[12] M.L. Baker, A. Bianchi, S. Carretta, D. Collison, R.J. Docherty, E.J.L. Mclnnes, A. McRobbie, C.A. Muryn, H. Mutka, S. Piligkos, M. Rancan, P. Santini, G.A. Timco, P.L.W. Tregenna-Piggott, F. Tuna, H.U. Gudel, R.E.P. Winpenny, Dalton Trans. 40, 2725 (2011).

[13] S. Carretta, P. Santini, G. Amoretti, M. Affronte, A. Ghirri, I. Sheikin, S. Piligkos, G. Timco, R.E.P. Winpenny, Phys. Rev. B 72, 060403(R) (2005).

[14] Y. Furukawa, K. Kiuchi, K. Kumagai, Y. Ajiro, Y. Narumi, M. Iwaki, K. Kindo, A. Bianchi, S. Carretta, G.A. Timco, R.E.P. Winpenny, Phys. Rev. B 78, 092402 (2008)

[15] P. Kozłowski, M. Antkowiak, G. Kamieniarz, J. Nanopart. Res. 13, 6093 (2011).

[16] P. Kozłowski, G. Kamieniarz, M. Antkowiak, F. Tuna, G.A. Timco, R.E.P. Winpenny, Polyhedron 28, 1852 (2009).

[17] M. Antkowiak, P. Kozłowski, G. Musiał, W. Florek, G. Kamieniarz, F. Esposito, Acta Phys. Pol. A 118, 965 (2010).

[18] P. Kozłowski, G. Kamieniarz, J. Nanosci. Nanotechnol. 11, 9175 (2011).

[19] T. Ślusarski, B. Brzostowski, D. Tomecka, G. Kamieniarz, Acta Phys. Pol. A 118, 967 (2010).

[20] T. Ślusarski, B. Brzostowski, D. Tomecka, G. Kamieniarz, J. Nanosci. Nanotechnol. 11, 9080 (2011).

[21] P. Sobczak, A. Barasiński, R. Matysiak, A. Drzewiński, G. Kamieniarz, A. Bieńko, J. Mrozinski, Acta Phys. Pol. A 118, 975 (2010).

[22] A. Tomkiewicz, A. Zygmunt, J. Mroziński, J. Mol. Struct. 644, 97 (2003).

[23] G. Kamieniarz, R. Matysiak, Comp. Mater. Sci. 28, 353 (2003).

[24] P. Sobczak, A. Barasiński, A. Drzewiński, G. Kamieniarz, J. Kłak, A. Bieńko, J. Mroziński, Polyhedron 28, 1838 (2009).

[25] A. Barasiński, P. Sobczak, A. Drzewiński, G. Kamieniarz, A. Bieńko, J. Mroziński, D. Gatteschi, Polyhedron 29, 1485 (2010).

[26] P. Ordejón, E. Artacho, J.M. Soler, Phys. Rev. B 53, R10441 (1996)

[27] J. van Slageren, R. Sessoli, D. Gatteschi, A.A. Smith, M. Helliwell, R.E.P. Winpenny, A. Cornia, A.L. Barra, A.G.M. Jansen, E. Rentschler, G.A. Timco, Chem. Eur. J. 8, 277 (2002).
[28] E.J.L. McInnes, S. Piligkos, G.A. Timco, R.E.P. Winpenny, Coord. Chem. Rev. 249, 2577 (2005).

[29] M. Affronte, S. Carretta, G.A. Timco, R.E.P. Winpenny, Chem. Commun. 18, 1789 (2007).

[30] O. Cador, D. Gatteschi, R. Sessoli, A.-L. Barra, G.A. Timco, R.E.P. Winpenny, J. Magn. Magn. Mater. 290-291, 55 (2005).

[31] F.K. Larsen, E.J.L. McInnes, H. El Mkami, J. Overgaard, S. Piligkos, G. Rajaraman, E. Rentschler, A.A. Smith, G.M. Smith, V. Boote, M. Jennings, G.A. Timco, R.E.P. Winpenny, Angew. Chem. Int. Ed. 42, 101 (2003).

[32] S. Piligkos, H. Weihe, E. Bill, F. Neese, H. El Mkami, G.M. Smith, D. Collison, G. Rajaraman, G.A. Timco, R.E.P. Winpenny, E.J.L. McInnes, Chem. Eur. J. 15, 3152 (2009)

[33] H.C. Yao, J.J. Wang, Y.S. Ma, O. Waldmann, W.X. Du, Y. Song, Y.Z. Li, L.M. Zheng, S. Decurtins, X.Q. Xin, Chem. Commun., 1745 (2006).

[34] N. Hoshino, M. Nakano, H. Nojiri, W. Wernsdorfer, H. Oshio, J. Am. Chem. Soc. 131, 15100 (2009).

[35] Spin Glass Theory and Beyond, Eds. M. Mézard, G. Parisi, M.A. Virasoro, Word Scientific, Singapore 1987.

[36] Introduction to Frustrated Magnetism, Eds. C. Lacroix, F. Mila, P. Mendels, Springer, Berlin 2010.

[37] J. Schnack, J. Low Temp. Phys. 142, 283 (2006).

[38] J. Schnack, Dalton Trans. 39, 4677 (2010).

[39] E. Lieb, T. Schultz, D.C. Mattis, Ann. Phys. (N.Y.) 16, 407 (1961)

[40] E. Lieb, D.C. Mattis, J. Math. Phys. 3, 749 (1962).

[41] D.M. Tomecka, V. Bellini, F. Troiani, F. Manghi, G. Kamieniarz, M. Affronte, Phys. Rev. B 77, 224401 (2008).

[42] V. Corradini, F. Moro, R. Biagi, V. De Renzi, U. del Pennino, V. Bellini, S. Carretta, P. Santini, V.A. Milway, G. Timco, R.E.P. Winpenny, M. Affronte, Phys. Rev. B 79, 144419 (2009).

[43] V. Bellini, A. Olivieri, F. Manghi, Phys. Rev. B $\mathbf{7 3}$, 184431 (2006).

[44] G. Kamieniarz, P. Kozłowski, G. Musiał, W. Florek, M. Antkowiak, M. Haglauer, A.C. D'Auria, F. Esposito, Inorg. Chim. Acta 361, 3690 (2008).

[45] K. Bernot, J. Luzon, R. Sessoli, A. Vindigni, J. Thion, S. Richeter, D. Leclercq, J. Larionova, A. van der Lee J. Am. Chem. Soc. 130, 1619 (2008).

[46] A. Barasiński, A. Drzewiński, G. Kamieniarz, Com put. Phys. Commun. 182, 2013 (2011). 